\title{
Sources and assumptions for the vicarious calibration of ocean color satellite observations
}

\author{
Sean W. Bailey, ${ }^{1,2, \star}$ Stanford B. Hooker, ${ }^{3}$ David Antoine, ${ }^{4}$ \\ Bryan A. Franz, ${ }^{1,5}$ and P. Jeremy Werdell ${ }^{1,6}$ \\ ${ }^{1}$ Ocean Biology Processing Group 614.8, NASA Goddard Space Flight Center Greenbelt, Maryland 20771, USA \\ ${ }^{2}$ Futuretech Corporation, 7307 Hanover Parkway, Suite D, Greenbelt, Maryland 20770, USA \\ ${ }^{3}$ NASA Goddard Space Flight Center, Greenbelt, Maryland 20771, USA \\ ${ }^{4}$ Laboratoire d'Océanographie de Villefranche (LOV) Observatoire Océanologique de Villefranche \\ (OOV) Quai de La Darse, BP 806238 Villefranche sur Mer Cedex France \\ ${ }^{5}$ SAIC, Inc., 4600 Powder Mill Road, Suite 400, Beltsville, Maryland 20705, USA \\ ${ }^{6}$ Science Systems and Applications, Inc, 10210 Greenbelt Road, Suite 600, Lanham, Maryland 20706, USA \\ ${ }^{*}$ Corresponding author: Sean.W.Bailey@ nasa.gov \\ Received 7 December 2007; accepted 25 February 2008; \\ posted 3 March 2008 (Doc. ID 90652); published 15 April 2008
}

\begin{abstract}
Spaceborne ocean color sensors require vicarious calibration to sea-truth data to achieve accurate waterleaving radiance retrievals. The assumed requirements of an in situ data set necessary to achieve accurate vicarious calibration were set forth in a series of papers and reports developed nearly a decade ago, which were embodied in the development and site location of the Marine Optical BuoY (MOBY). Since that time, NASA has successfully used data collected by MOBY as the sole source of sea-truth data for vicarious calibration of the Sea-viewing Wide field-of-view Sensor (SeaWiFS) and Moderate Resolution Imaging Spectroradiometer instruments. In this paper, we make use of the 10-year, global time series of SeaWiFS measurements to test the sensitivity of vicarious calibration to the assumptions inherent in the in situ requirements (e.g., very low chlorophyll waters, hyperspectral measurements). Our study utilized field measurements from a variety of sources with sufficient diversity in data collection methods and geophysical variability to challenge those in situ restrictions. We found that some requirements could be relaxed without compromising the ability to vicariously calibrate to the level required for accurate water-leaving radiance retrievals from satellite-based sensors. (C) 2008 Optical Society of America

OCIS codes: $\quad 010.0010,280.0280,120.0120,010.4450,120.5630$.
\end{abstract}

\section{Introduction}

The accurate estimation of water-leaving radiance, $L_{W}(\lambda)$, from spaceborne radiometers requires vicarious calibration, which is a system-level process that accounts for systematic biases in the atmospheric correction algorithm and changes to the prelaunch calibration resulting from the transfer to orbit. Gordon [1] and Clark et al., [2,3] proposed guidelines (Table 1 ) for a successful vicarious calibration activity using in situ

0003-6935/08/122035-11 $\$ 15.00 / 0$

(C) 2008 Optical Society of America data as sea truth. Briefly, they suggest that data be collected: in low chlorophyll waters with ocean optical properties horizontally homogeneous over several kilometers; under clear atmospheric conditions (i.e., maritime aerosols, aerosol optical thickness at $865 \mathrm{~nm}, \tau_{a}(865 \mathrm{~nm})$, less than 0.10$)$; using hyperspectral instruments to allow the individual response functions for the satellite channels to be applied; and, with extraordinarily well-characterized and calibrated field instruments to minimize the sources of uncertainty contributed by the sea-truth observations (e.g., stray light characterization). 
The Ocean Biology Processing Group (OBPG) currently uses Marine Optical Buoy (MOBY) data [2] in the vicarious calibration of the Sea-viewing Wide Field-of-view Sensor (SeaWiFS) and the NASA Moderate Resolution Imaging Spectroradiometer (MODIS) [4]. The location of MOBYand its instrumentation were intended to comply with the a priori requirements listed above [1, 3$]$. However, the maintenance of MOBY requires a well-coordinated, dedicated program and the possibility exists that it, or similar instruments with sufficient financial and human resources, will not be available for future missions (e.g., the Visible/Infrared Imager/Radiometer Suite (VIIRS) [5], scheduled to launch no sooner than 2010). This possibility, along with the need to vicariously calibrate future missions rapidly after launch, necessitates that alternative sources of sea-truth data be explored. Werdell et al. [6] demonstrated the feasibility of using an ocean surface reflectance model for determining the target $L_{W}(\lambda)$ for vicarious calibration. While a model-based solution may suffice, measured radiances remain desirable, because a model, however sophisticated, cannot capture all of the variability present in the natural marine environment.

It was originally suggested that the vicarious calibration of the satellite sensor be accomplished using dedicated ship-based measurements made shortly after launch when routine on-orbit data collection commenced [1,2]. The long-term stability of the calibration derived using data collected during these initialization cruises would then be continuously monitored using a moored radiometer located at a site where the atmospheric conditions are well understood and easily modeled so as to have minimal impact on the retrieval of $L_{W}(\lambda)$ from the spaceborne instrument. Initialization cruises were undertaken following the launch of SeaWiFS and both MODIS instruments (aboard the Terra and Aqua spacecraft); however, the data collected on these cruises were ultimately used for validation, not vicarious calibration. When vicarious calibration was first implemented for SeaWiFS with the first reprocessing in January 1998, MOBY was used as the sole source for sea-truth data [7].

In this paper, the efficacy of alternative field measurements for vicarious calibration is examined and the $a$ priori assumptions for the collection of these field measurements are evaluated using the 10-year SeaWiFS mission as a test case. A number of highquality optical measurements whose calibration, sampling, and processing procedures adhere to the community-vetted ocean optics protocols [8] exist for this analysis including the above- and in-water shipboard in situ data from the NASA bio-Optical Marine Algorithm Data set (NOMAD) [9] and the in-water radiometric mooring data from the Bouée pour l'acquisition de Séries Optiques à Long Terme (BOUSSOLE) project [10]. There are additional data sources (e.g., the SeaWiFS Photometer Revision for Incident Surface Measurement (SeaPRISM) [11]; the Satellite validation for Marine biology and
Aerosol Determination radiometers (SIMBAD), and the Advanced SIMBAD (SIMBADA) [12]) that were not considered because of a lack of one or more of the SeaWiFS bands or availability of the data.

This assemblage of sea-truth measurements, with a diverse geographic extent (Fig. 1) and varied environmental conditions, not only allows for the examination of these data as alternative vicarious calibration data sources, but also permits testing of the assumptions (Table 1) as to the requirements of a vicarious calibration data set. In this paper, these seatruth measurements were used to derive the average vicarious calibration coefficients, $\bar{g}^{\prime}$, for each SeaWiFS visible band and evaluate these against the standard MOBY-derived calibration, $\bar{g}$. Additionally, in an effort to evaluate the impact of using a multispectral instrument in lieu of a hyperspectral one for vicarious calibration, the spectral resolution of the MOBY data was also degraded to simulate a fixed-wavelength instrument with $10 \mathrm{~nm}$ bandwidths centered on the nominal SeaWiFS wavelengths.

\section{Satellite Data}

The SeaWiFS mission provides a 10-year time series of global ocean color data. The instrument is well characterized and its stability over the course of the mission is well documented $[7,13,14]$. The majority of SeaWiFS data are at a nominal $4.5 \mathrm{~km}$ spatial resolution. This global area coverage (GAC) data are subsampled from full-resolution data (nominally $1.1 \mathrm{~km}$ at nadir) with every fourth pixel of a scan line and every fourth scan line being recorded. SeaWiFS has a limited capacity for storing full-resolution local area coverage (LAC) data on the flight data recorder for subsequent downlink, and it also transmits the full LAC dataset to local ground stations via direct broadcast. Until December 2004, a global network of ground stations provided near-global LAC coverage. For 2005 and onward, the only consistently available LAC coverage is of the U.S. coast. For purposes of calibration, only the LAC resolution data are used to ensure that close proximity to clouds or other potential sources of stray light can be properly identified and screened.

Given that this work addresses the use of both hyperand multispectral data for use in vicarious calibration, a brief review of the SeaWiFS spectral response is prudent. The SeaWiFS instrument is a filter-based radiometer that measures top-of-atmosphere (TOA)



Fig. 1. Map showing the locations for the in situ data used in this study. 
Table 1. Current Recommended Requirements on Sea-Truth Data for Vicarious Calibration Activities ${ }^{a}$

\begin{tabular}{llccc}
\multicolumn{1}{c}{ Requirement } & Ref. & MOBY & $\begin{array}{c}\text { COTS } \\
\text { Mooring }\end{array}$ & $\begin{array}{c}\text { Free-fall } \\
\text { Radiometer }\end{array}$ \\
\hline (1) clear maritime atmosphere & 1 & $\checkmark$ & $\checkmark$ & $\checkmark$ \\
(2) clear-water site & $1,2,3$ & $\checkmark$ & $\checkmark$ & $\checkmark$ \\
(3) horizontally homogeneous water mass & 1 & $\checkmark$ & $\checkmark$ & $\checkmark$ \\
(4) hyperspectral instrumentation & 2,3 & $\checkmark$ & $\checkmark$ & $\checkmark$ \\
(5) extraordinary calibration & 3 & $\checkmark$ & $\checkmark$ \\
(6) daily-to-weekly monitoring of derived $L_{\text {wn }}$ & 3 & $\checkmark$ & $\checkmark$ \\
(7) avoidance of platform perturbation & 3 & $\checkmark$ & $\checkmark$ \\
(8) cloud-free site & 3 & $\checkmark$ & $\checkmark$ \\
(9) coincident aerosol measurements & $1,2,3$ & & $\checkmark$ \\
(10) atmosphere free of terrestrial influence & 1,3 & $\checkmark$ & $\checkmark$ & $\checkmark$ \\
(11) free from biofouling & 3 & & $\checkmark$ \\
\hline
\end{tabular}

${ }^{a}$ Requirements for vicarious calibration determined a priori with associated reference(s). Checks indicate in situ data source compliance.

radiance in six visible and two near infrared (NIR) bands. Prior to launch, the spectral response functions for each band were measured and several bands were found to exhibit between $1-4 \%$ out-of-band response, which results in a significant radiance contribution from wavelengths outside the nominal bandpass [15]. The atmospheric correction algorithm considers the full spectral response of the instrument [16]. Thus, without subsequent correction, the retrieved $L_{W}(\lambda)$ correspond to the full spectral bandpass of each band. Under the operational data processing for SeaWiFS, an out-of-band correction $[17,18]$ is applied. This correction adjusts the full-resolution spectral bands to equivalent $10 \mathrm{~nm}$ bands for ease of comparison to in situ measured radiances, which are typically narrow-band, multispectral measurements.

\section{Sea-Truth $L_{w}$ Data}

The principal input variable for the vicarious calibration procedure is in situ $L_{W}$. An $L_{W}$ data set collected using diverse instrumentation and deployment methods with the necessary spectral resolution to vicariously calibrate the visible bands for SeaWiFS was assembled, as described below. The adherence to a well-documented set of data collection and processing protocols allow these data to be extended beyond their original intent. It is hypothesized that these data are of sufficient quality to be used for vicarious calibration in the absence of a MOBY-grade instrument, although most were not collected for that specific purpose. Table 1 lists the requirements for vicarious calibration to be examined in this paper and indicates which of these requirements are met by the various sea-truth data sources.

\section{A. Marine Optical Buoy-MOBY}

The National Oceanic and Atmospheric Administration (NOAA) maintains MOBY at a site approximately $20 \mathrm{~km}$ west of the Hawaiian island of Lanai. The mooring has been collecting measurements of up- and down-welled radiance at this clear-water site since 1996. MOBY provides subnanometer $(0.6 \mathrm{~nm})$ spectral resolution from $350-955 \mathrm{~nm}$, and thus provides the capability to match the spectral response of each of the eight SeaWiFS bands. The MOBY
Operations Team (MOT) maintains the calibration of MOBY, working closely with members of the National Institute for Standards and Technology (NIST) [19]. The location of the site was selected specifically to meet the environmental conditions set forth in the requirements for vicarious calibration (Table 1 ). The MOT-provided bandpass convolved $L_{W}$ values are used as the baseline for this study, as they are assumed to be the most accurate values for comparison with the satellite retrievals. In addition, the fullresolution hyperspectral $L_{u}$ and $E_{d}$ data for MOBY were acquired and subsampled to mimic a multispectral instrument. This was done to allow a direct test of the hyperspectral requirement [1-3]. While both the NOMAD and BOUSSOLE data sets use multispectral, commercial off-the-shelf (COTS) instrumentation, neither provides for a direct comparison to the standard MOBY measurements as they introduce a number of differences (e.g., environmental factors) in the process that would complicate the hyper-versus multispectral analysis.

The multispectral MOBY (msMOBY) $L_{u}$ and $E_{d}$ data were output as $10 \mathrm{~nm}$ bandpass averages centered on the nominal SeaWiFS wavelengths $(\lambda-5 \mathrm{~nm} \leq \lambda<\lambda+5 \mathrm{~nm})$, which were then processed to $L_{W}$ following the method used by the MOT [3]. The exclusion criteria for the in situ data as described in [4] were applied to the msMOBY data prior to use for the vicarious calibration. In the end, only the scenes included in the calculation of the vicarious calibration coefficients from the standard MOBY set were used. This provides a one-to-one correspondence between the hyper- and multispectral MOBY data sets.

\section{B. NOMAD}

The NASA OBPG generated a standalone in situ data set of remote-sensing relevant products, NOMAD [9], using the data archived in the SeaWiFS Biooptical Archive and Storage System (SeaBASS) [20]. NOMAD consists of well-screened, consistently processed coincident surface observations of radiometric spectra, bulk water temperatures and salinities, chlorophyll $a\left(C_{a}\right)$, and associated metadata that were collected by NASA, other agencies, and university research scientists in support of ocean color 
activities with adherence to well-established deployment and processing protocols [8]. It is publicly available for the purpose of expediting community bio-optical algorithm development and facilitating satellite calibration and validation activities. Since NOMAD was designed for such purposes, the majority of the data have values for the six visible bands of SeaWiFS. Data without all six visible bands were excluded from this analysis.

\section{BOUSSOLE}

The BOUSSOLE project [21] maintains a mooring located in the Ligurian sea, a sub-basin of the Western Mediterranean sea. The project provides a long-term time series of optical properties in support of calibration and validation activities associated with satellite ocean color missions and bio-optics research and algorithm development.

The buoy, which largely replicates the MOBY approach with the use of COTS instrumentation [10], provides above-water measurements of surface irradiance and in-water $(4$ and $9 \mathrm{~m})$ measurements of the upward and downward planar irradiance, upwelled radiance at nadir, chlorophyll fluorescence, and beam attenuation $(660 \mathrm{~nm})$. At the deepest measurement depth $(9 \mathrm{~m})$, backscattering at 443 and $560 \mathrm{~nm}$, conductivity, temperature, and pressure also are recorded. The wavelengths for the apparent optical property measurements are $412,443,490,510$, 560,665 , and $683 \mathrm{~nm}$.

Early deployments of the BOUSSOLE mooring exhibited an odd behavior for the $412 \mathrm{~nm}$ band. Unfortunately, the original mooring was lost at sea, so the underlying cause for the odd $412 \mathrm{~nm}$ data was never resolved. These data were excluded from the computation of the gain, but only for the $412 \mathrm{~nm}$ band, as the problem did not appear to affect the other bands. The BOUSSOLE data set was limited to the period when SeaWiFS LAC resolution data was available to NASA (prior to 24 December 2004).

\section{In Situ Source Comparison}

The BOUSSOLE and NOMAD data sets offer a broad dynamic range for both radiance measurements and $C_{a}$ concentrations compared to that of the MOBY data set (Fig. 2). The waters around MOBYare consistently oligotrophic, with low $C_{a}$, nearly constant $L_{W}(555)$, and only a moderately variable $L_{W}(443)$ (as modulated by $C_{a}$ ). In contrast, both the BOUSSOLE and NOMAD data sets exhibit large variability, with the NOMAD set having the greatest variability of the three. While the in-water optical property differences among the three data sets are large, the atmospheric properties are largely consistent. The $\tau_{a}(865)$ measured over the three data sets have very similar distributions dominated by low aerosol load conditions.

\section{Methods}

\section{A. Vicarious Calibration}

The total radiance measured at TOA $\left(L_{t}(\lambda)\right)$ can be viewed as the sum of various radiance components:
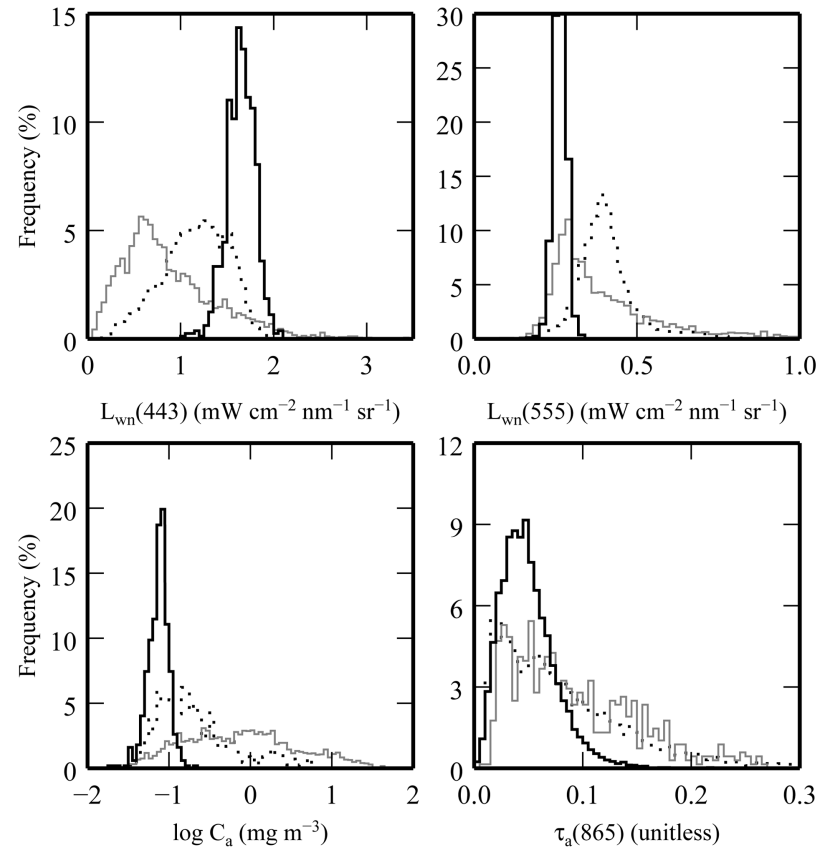

Fig. 2. Frequency distributions for key parameters of the in situ data sets. Data from MOBY are shown by the solid black curve, BOUSSOLE by the dotted black curve, and NOMAD by the solid gray curve. The $C_{a}$ data for MOBY and the $\tau_{a}(865)$ data for NOMAD are coincident SeaWiFS $C_{a}$ retrievals.

$$
\begin{aligned}
L_{t}(\lambda)= & {\left[L_{r}(\lambda)+L_{a}(\lambda)+t_{d_{v}}(\lambda) L_{f}(\lambda)\right.} \\
& \left.+t_{d_{v}}(\lambda) L_{W}(\lambda)\right] t_{g_{v}}(\lambda) t_{g_{s}}(\lambda),
\end{aligned}
$$

where $L_{r}(\lambda), L_{a}(\lambda)$, and $L_{f}(\lambda)$ represent the radiance contributions associated with Rayleigh scattering $[22,23]$, aerosols (including Rayleigh-aerosol interactions) [24], and surface whitecaps [25-27], respectively. The $t_{d_{v}}(\lambda)$ term accounts for diffuse transmittance along the sensor view path from the surface to the satellite. Gaseous absorption in the Sun to surface and surface to sensor paths are accounted for with the $t_{g_{s}}(\lambda)$ and $t_{g_{v}}(\lambda)$ terms [24,28]. The effects of polarization are ignored here as the SeaWiFS instrument includes a polarization scrambler that minimizes the impact of polarization on the radiance measurements. Specular glint radiance is also ignored as cases where sun glint affects the scene are explicitly excluded from any calibration data set.

In the forward process of the atmospheric correction algorithm, the desired quantity is $L_{W}(\lambda)$. To perform a vicarious calibration, this unknown quantity is replaced by $L_{W}(\lambda)$ values from the sea-truth measurements, and a vicarious $L_{t}(\lambda)$ is computed. In essence, the atmospheric correction algorithm is inverted to retrieve TOA radiance from an input $L_{W}(\lambda)[4]$.

The resulting $L_{t}(\lambda)$ is compared to the satellite measured $L_{t}(\lambda)$ and a gain coefficient, $g_{\lambda}$, is derived that would force agreement of the measured $L_{t}(\lambda)$ and the vicarious $L_{t}(\lambda)$ : 


$$
g_{\lambda}=\frac{L_{t}^{\text {vicarious }}(\lambda)}{L_{t}^{\text {measured }}(\lambda)}
$$

The final $\overline{g_{\lambda}}$ is determined as

$$
\left[\sum_{j=1}^{N} g_{\lambda}(j)\right] / N,
$$

where the index $j$ refers to the individual $g_{\lambda}$ values in the semi-interquartile range of all $g_{\lambda}$ and $N$ is the number of calibration points. This study focuses only on the vicarious calibration of the visible bands. The vicarious calibration of the NIR bands is assumed fixed and set to the operational gain values.

For each in situ record, the corresponding LAC resolution satellite data file was identified. A nominal $1^{\circ} \times 1^{\circ}$ box centered on the in situ location was extracted from the full satellite file. This extracted satellite file was processed as described in [4] to produce $g_{\lambda}$. In addition, satellite-derived $C_{a}, \tau_{a}(8 \overline{6} 5 \mathrm{~nm})$, and the Ångstrøm exponent at $510 \mathrm{~nm}$ were retrieved.

Following [4], the exclusion criteria applied to the data as the baseline for this study include: $C_{a}$ concentration of less than $0.2 \mathrm{mg} \mathrm{m}^{-3}, \tau_{a}(865)$ of less than 0.15 , solar zenith angle of less than $70^{\circ}$, sensor zenith angle of less than $56^{\circ}$, a coefficient of variation for the scene of less than 0.10 , and the requirement that all 25 pixels in the $5 \times 5$ pixel box centered on the in situ data be valid (i.e., not flagged as any of the following: cloud, stray light, high light, cloud shadow, land, glint, or navigation warning or failure). It should be noted that these criteria are more stringent than those employed in the validation of remotely sensed ocean color data products [29].

\section{Nonnegligible NIR Water-Leaving Radiance}

A primary assumption of the Gordon and Wang [24] atmospheric correction algorithm is that the ocean does not contribute to the TOA signal in the NIR, (i.e., all radiance reaching the sensor is of atmospheric origin). Using this assumption, the NIR bands can then be employed for the aerosol determination in the atmospheric correction process. This assumption holds for low $C_{a}$, Case 1 waters where phytoplankton are the only optically significant water column contributor [30]. However, not all the in situ data in our NOMA $\bar{D}$ or BOUSSOLE data sets were collected in low $C_{a}$, Case 1 waters. This complicates the vicarious calibration of the visible bands, as water-leaving radiance in the NIR bands cannot be assumed negligible and must be known or estimated [18,31]. Naturally, errors in the estimate of the aerosol contribution will negatively affect the vicarious calibration.

A model [18] is employed by the OBPG processing code, MSl12, to estimate $L_{W}$ (NIR) in forward processing, and this same model was applied to the in situ data to obtain an estimate of the $L_{W}$ (NIR) contribution for the vicarious calibration. The model requires as input both $C_{a}$ and the remote-sensing reflectance at
555 and $670 \mathrm{~nm}$ :

$$
R_{\mathrm{rs}}(\lambda)=\frac{L_{W}(\lambda)}{E_{s}(\lambda)}
$$

where $E_{s}$ is the downwelling irradiance at the sea surface. Where in situ $C_{a}$ was not available, it was estimated from the in situ radiances using the OC2 (ocean chlorophyll) algorithm [32], where $C_{a}$ is statistically correlated to the ratio of $R_{\mathrm{rs}}(490 \mathrm{~nm})$ to $R_{\mathrm{rs}}(555 \mathrm{~nm})$. As the input $C_{a}$ only modulates the estimated total absorption term in the model, the use of a model estimated $C_{a}$ does not significantly impact the retrieved NIR radiance in Case 2 or high $C_{a}$ Case 1 waters. Incorporation of the $L_{W}(\mathrm{NIR})$ correction into the vicarious calibration process allows for $g^{\prime}$ to be derived from waters with higher $C_{a}$ concentrations than would otherwise be possible. The vicarious calibration process described by [4] assumes $L_{W}(\mathrm{NIR})$ is negligible. A modification to MSL12 was necessary to allow $L_{W}(\mathrm{NIR})$ to be input in the vicarious calibration inversion process.

\section{Results and Discussion}

\section{A. Baseline Comparison}

As a first step, each data set was evaluated using the same criteria applied to the MOBY-derived $g_{\lambda}$ data set (Table 2). For this initial comparison the assumption of negligible $L_{W}(\mathrm{NIR})$ was made. For the NOMAD data set no modifications to this criteria set were required to obtain a sufficient number (i.e., $N \geq 40$ ) for statistical confidence [4]. However, of the 1039 in situ measurements with coincident SeaWiFS LAC coverage, only 449 pass the valid pixel criteria; 189 of these pass the chlorophyll threshold criteria, while 125 fail to pass the aerosol optical thickness or geometry constraints. Only 64 pass all of the default calibration exclusion criteria.

For the BOUSSOLE data set, the short time for which the buoy was deployed and SeaWiFS LAC coverage was readily available limits the number of coincident scenes to 76. After applying the exclusion criteria, it was determined that a minor increase in the $C_{a}$ threshold from 0.2 to $0.25 \mathrm{mg} \mathrm{m}^{-3}$ was necessary to increase the valid sample size for $g_{\lambda}^{\prime}$ to greater than 40 .

As depicted in Fig. 3 , the $\bar{g}_{\lambda}^{\prime}$ for all sources fall within 1 standard deviation of the $g_{\lambda}$ for all bands. As a measure of the variance in $g_{\lambda}$, a $2 \sigma$ coefficient of variation $(\mathrm{CV})$ was computed as

$$
\mathrm{CV}_{\lambda}=100 * \frac{2 * \sigma_{\lambda}}{\bar{g}_{\lambda}}
$$

These values are reported in Table 2 . To quantify how well the $\bar{g}_{\lambda}^{\prime}$ derived from the alternative sources compared to the $\bar{g}_{\lambda}$ derived from MOBY, an unbiased percent difference (UPD) was computed: 
Table 2. Vicarious Gain Coefficients for Standard Method ${ }^{a}$

\begin{tabular}{|c|c|c|c|c|c|c|c|}
\hline Source & $\mathrm{N}$ & 412 & 443 & 490 & 510 & 555 & 670 \\
\hline$(\sigma)$ & & $(0.009)$ & $(0.009)$ & $(0.008)$ & $(0.009)$ & $(0.009)$ & $(0.007)$ \\
\hline NOMAD & 64 & 1.0395 & 1.0135 & 0.9967 & 0.9962 & 0.9989 & 0.9693 \\
\hline$(\sigma)$ & & $(0.013)$ & $(0.013)$ & $(0.014)$ & $(0.017)$ & $(0.013)$ & $(0.009)$ \\
\hline UPD & & 0.1300 & 0.01480 & 0.2464 & -0.1003 & -0.0200 & -0.1854 \\
\hline BOUSSOLE $^{b}$ & 46 & $1.0402^{c}$ & 1.0129 & 0.9961 & 1.0015 & 1.0007 & 0.9672 \\
\hline UPD & & 0.1637 & -0.0148 & 0.2163 & 0.1650 & 0.0700 & -0.2938 \\
\hline
\end{tabular}

${ }^{a}$ Gain coefficients using the threshold criteria defined in [4]. The standard deviations are shown in parentheses. ${ }^{b} C_{a}$ threshold increased to $0.25 \mathrm{mg} \mathrm{m}^{-3}$ for the BOUSSOLE data set to bring the $\bar{N}$ to a minimum of $40{ }^{c}$ The $412 \mathrm{~nm}$ data for BOUSSOLE used only 9 points.

$$
\mathrm{UPD}_{\lambda}=100 * \frac{\bar{g}_{\lambda}^{\prime}-\left(\bar{g}_{\lambda}^{\prime}+\bar{g}_{\lambda}\right) / 2}{\left(\bar{g}_{\lambda}^{\prime}+\bar{g}_{\lambda}\right) / 2}=100 * \frac{\bar{g}_{\lambda}^{\prime}-\bar{g}_{\lambda}}{\bar{g}_{\lambda}^{\prime}+\bar{g}_{\lambda}}
$$

The $\mathrm{CV}_{\lambda}$ for MOBY ranged between 1.4 and $1.8 \%$. The largest $\mathrm{UPD}_{\lambda}$ for the alternative sources was $0.29 \%$, well within the MOBY $\mathrm{CV}_{\lambda}$. These results demonstrate that alternative data sources are capable of providing $\bar{g}$ that are statistically equivalent to that provided by MOBY.

\section{B. Hyperspectral versus Multispectral Resolution}

The vicarious gain coefficients obtained using the multispectral MOBY data set are listed in Table $\underline{3}$, along with the relative percent difference from the standard MOBY-derived $\bar{g}$. Relative percent difference (RPD) was calculated as

$$
\mathrm{RPD}_{\lambda}=100 * \frac{\bar{g}_{\lambda}^{\prime}-\bar{g}_{\lambda}}{\bar{g}_{\lambda}}
$$

RPD was used in this case, unlike the UPD used for the comparisons for the alternative sources, as here it was assumed that the hyperspectral MOBY values are truth. All of the vicarious gain coefficients derived from this msMOBY data set are within $0.45 \%$ of the standard MOBY-derived coefficients. The two bands with the most significant out-of-band response for SeaWiFS are the 510 and $555 \mathrm{~nm}$ bands [17]. Both of these bands show a negative bias relative to the standard MOBY-derived coefficients. This result is expected because the out-of-band response in these bands would serve to increase the observed radiance above the measurement for the nominal band. The nearly equivalent positive biases seen

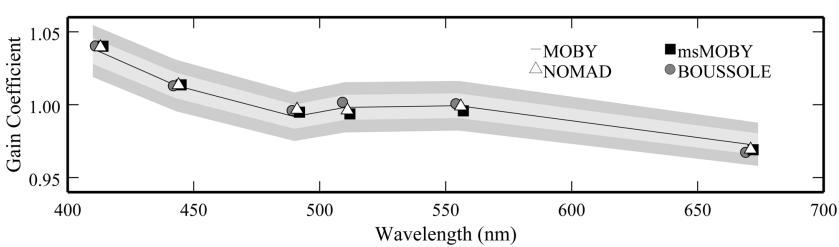

Fig. 3. Vicarious calibration coefficients as a function of wavelength. The standard MOBY-derived $\overline{g_{\lambda}{ }^{\prime}}$ (solid curve) are overplotted by the msMOBY-, NOMAD-, and BOUSSOLE-derived $\bar{g}_{\lambda}{ }^{\prime}$. The shaded regions indicate the ranges for the first (light-gray) and second (dark-gray) standard deviations of the mean for $\overline{g_{\lambda}}$. for bands 412 and 490 were unexpected, as these bands do not have a large out-of-band response.

In a sense, SeaWiFS can be viewed as a worst-case scenario with respect to the out-of-band response. MODIS does not have the same level of out-of-band response seen in SeaWiFS [33], and it is expected that future instruments will also have a small outof-band response. Given that the results presented here show the msMOBY-derived $\bar{g}^{\prime}$ to be within the variation exhibited by the standard MOBY-derived $\bar{g}$, the uncertainty introduced by using multispectral instruments in lieu of a hyperspectral radiometer for vicarious calibration is at an acceptable level.

\section{C. $\quad C_{a}$ Threshold}

Using in situ data with a broad dynamic range of $C_{a}$ concentrations allows us to test the requirement for low $C_{a}$ waters for vicarious calibration. Vicarious calibration coefficients derived from the NOMAD and BOUSSOLE data sets were computed after removal of model-estimated $L_{W}$ from the NIR bands. These NIR-corrected vicarious calibration coefficients were compared to the coefficients derived from the default method (i.e., without prior removal of nonnegligible NIR radiance). Figure 4 shows that nonnegligible NIR radiance has an impact on data with a $C_{a}$ concentration above about $0.5-0.7 \mathrm{mg} \mathrm{m}^{-3}$. The NIR-corrected data set shows that, for higher $C_{a}$ concentrations, accounting for the non-negligible $L_{W}(\mathrm{NIR})$ can bring the derived vicarious calibration coefficient in line with those derived from lower concentration waters, at least up to $1-3 \mathrm{mg} \mathrm{m}^{-3}$.

\section{D. $\tau_{a}$ Threshold}

For all of the data sets used in this study, the satellite-derived $\tau_{a}(865 \mathrm{~nm})$ was in the range of $0.01-$ 0.205 (Fig. 5). For this range, there does not appear to be any trend in the derived $\bar{g}^{\prime}$. For this analysis, no

Table 3. Vicarious Gain Coefficients Using msMOBY Data ${ }^{a}$

\begin{tabular}{rccrrrr}
\hline & 412 & 443 & 490 & \multicolumn{1}{c}{510} & \multicolumn{1}{c}{555} & \multicolumn{1}{c}{670} \\
\hline & 1.0401 & 1.0136 & 0.9949 & 0.9937 & 0.9958 & 0.9691 \\
RPD & 0.3183 & 0.0395 & 0.3126 & -0.4508 & -0.3502 & -0.3906 \\
\hline
\end{tabular}

${ }^{a}$ Gain coefficients for MOBY data processed to mimic a multispectral, COTS instrument. 

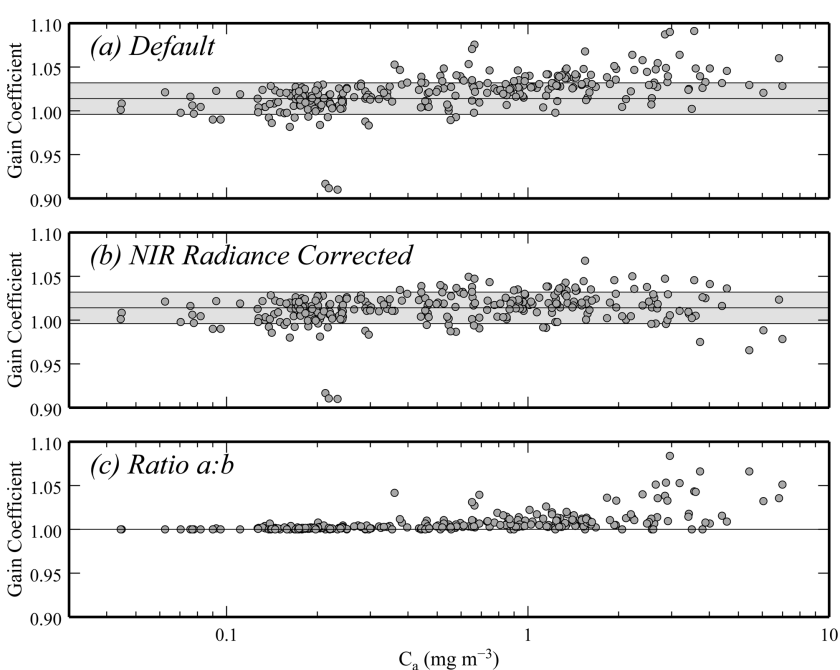

Fig. 4. $g(443 \mathrm{~nm})$ as a function of satellite-derived $C_{a}$ for (a) NIRuncorrected data set, (b) the NIR-corrected data set and the ratio of the two, and (c) ratio of (a) to (b).

restriction was placed on $\tau_{a}(865 \mathrm{~nm})$ and $C_{a}$ concentration was limited to $3 \mathrm{mg} \mathrm{m}^{-3}$, with correction for nonnegligible $L_{W}(\mathrm{NIR})$ applied. The requirement that $\tau_{a}(865 \mathrm{~nm})$ be $\leq 0.10$ [1] is met by the vast majority of the data used in this study. For the cases that exceed 0.10 , the vicarious calibration coefficients do not show any trend or bias relative to the low aerosol cases. As the majority of the data used in this study have $\tau_{a}(865 \mathrm{~nm})<0.15$, no statement can be made as to the impact that larger $\tau_{a}(865 \mathrm{~nm})$ may have on the gain estimation.

\section{E. Impact of Aerosol Type}

The wide geographic distribution for the various target data sets allows for the examination of vicarious calibration coefficients versus satellite-retrieved aerosol type, as identified by the Ångstrøm exponent between 510 and $865 \mathrm{~nm}$. Again, no restriction was placed on $\tau_{a}(865 \mathrm{~nm})$ and $C_{a}$ concentration was allowed to reach to $3 \mathrm{mg} \mathrm{m}^{-3}$. As is evident from Fig. 6 , the variation for the data sets covers the range of Angstrøm exponents possible given the aerosol models used in the atmospheric correction process. For the MOBY set, there are few points with an Ångstrøm exponent greater than 0.75 ; the majority fall below 0.5 and are thus representative of maritime aerosols. For the NOMAD and BOUSSOLE data sets, there is nearly an equal amount of points with an Ångstrøm

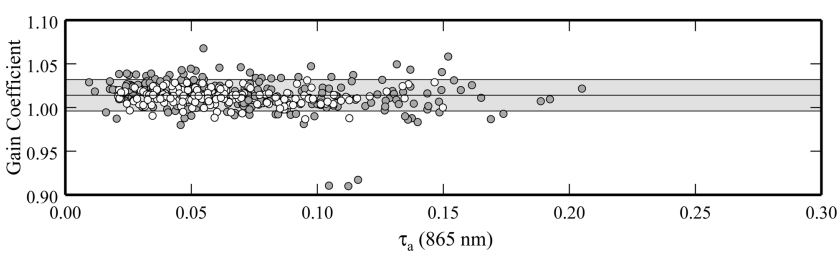

Fig. 5. $g^{\prime}(443 \mathrm{~nm})$ as a function of the satellite-estimated $\tau_{a}(865 \mathrm{~nm})$. Data from MOBY (open circles), as well as the NIRcorrected NOMAD and BOUSSOLE data sets (filled circles) are shown. exponent higher than 0.5 as lower. The lack of a trend in the $\bar{g}^{\prime}$ may be because, even with the higher Ångstrøm exponent data, the $\tau_{a}(865 \mathrm{~nm})$ are generally less than 0.15 (see Fig. 5). It is apparent from Fig. 6 that the satellite-retrieved aerosol type does not impact the retrieved vicarious calibration coefficients, at least for the data sets used in this study.

\section{F. Validation Comparisons}

To assess the impact of using the gains computed from the NOMAD and BOUSSOLE data sets on the satellite-derived products, a validation analysis was performed [29]. The SeaWiFS data products were reprocessed first by using the msMOBY-derived vicarious gain coefficients (Table 3) and then by using a weighted average of the NOMAD and BOUSSOLE coefficients (Table 4). The results were compared to the standard MOBY-derived validation data set for the deep water subset.

For both the msMOBY and NOMAD/BOUSSOLE validation runs, the satellite data were processed with the out-of-band correction [17] disabled. This correction was intended to produce nominal $10 \mathrm{~nm}$ bandpass values for the SeaWiFS $L_{W}$ data, given that the MOBY data used to derive the vicarious coefficients include the full spectral response for each band. The msMOBY, NOMAD, and BOUSSOLE data do not replicate the full spectral response of the satellite sensor, so it is assumed that any out-of-band effect is absorbed into the gains derived from these multispectral data sets.

As presented in Table 5 , the validation results for all three cases are in good agreement, within $\pm 1.5 \%$ (in absolute UPD) for all normalized water-leaving radiance retrievals $\left(L_{\mathrm{WN}}\right)$ except the $670 \mathrm{~nm}$ band. Relative to the MOBY-derived gains set, the largest deviation of the median ratio for the msMOBY validation set is $1.2 \%$ at $490 \mathrm{~nm}$ (ignoring $670 \mathrm{~nm}$ ). For the combination NOMAD/BOUSSOLE the largest deviation of the median ratio is $3.35 \%$ at $510 \mathrm{~nm}$. Overall, the smallest deviation from unity, (i.e., smallest combined bias with respect to in situ) for all wavelengths is for the NOMAD/BOUSSOLE gain set. However, for this gain set the band ratio $C_{a}$ algorithm product exhibits a high bias relative to both the in situ and the validation comparisons for both MOBY-derived gain sets. This is due to the spectral inconsistency (signed differences) between the 443 and $555 \mathrm{~nm}$ bands, which impacts the $R_{\mathrm{rs}}$ ratios used as input into the algorithm. While the NOMAD/BOUSSOLE results for

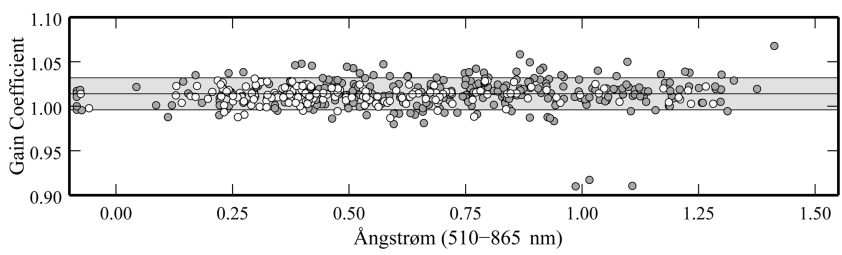

Fig. 6. $g(443 \mathrm{~nm})$ derived from MOBY (open circles), as well as the NIR-corrected $g^{\prime}(443 \mathrm{~nm})$ derived from NOMAD and BOUSSOLE data sets (filled circles) versus the satellite-estimated Ångstrøm (510 : $865 \mathrm{~nm})$ exponent. 
Table 4. Combined NOMAD and BOUSSOLE Vicarious Gain Coefficients $^{a}$

\begin{tabular}{lcccccc}
\hline $\mathrm{N}$ & 412 & 443 & 490 & 510 & 555 & 670 \\
\hline 110 & 1.0396 & 1.0133 & 0.9964 & 0.9984 & 0.9997 & 0.9684 \\
\hline
\end{tabular}

${ }^{a}$ Weighted average of the NOMAD- and BOUSSOLE-derived vicarious calibration coefficients.

$L_{\mathrm{WN}}(555 \mathrm{~nm})$ are closer to unity with respect to the in situ data, the $L_{\mathrm{WN}}(443 \mathrm{~nm})$ comparisons do not show the same level of improved agreement. Thus, while overall the bias is less for validation using this gain set, the relative spectral bias is larger between these two bands (e.g.,. larger difference in ratio), resulting in a slightly biased result for the derived products. The relative impact of this spectral bias can be seen in Fig. 7. For both the NOMAD/BOUSSOLE and msMOBY alternative $\bar{g}^{\prime}(\lambda)$ sets, the satellite-derived chlorophyll values for the validation data set are biased high for the oligotrophic range $\left(0-0.10 \mathrm{mg} \mathrm{m}^{-3}\right)$, biased low for the moderate eutrophic range $\left(1.0-3.0 \mathrm{mg} \mathrm{m}^{-3}\right)$, and exhibit almost no bias in the mesotrophic range $\left(0.10-1.0 \mathrm{mg} \mathrm{m}^{-3}\right)$ relative to satellite chlorophyll estimated using the standard MOBY-derived $\bar{g}(\lambda)$. Despite this obvious spectral bias effect, the relative difference in the resulting chlorophyll retrievals are well within the accepted uncertainty of $\pm 35 \%$ [34].

The validation results for $L_{\mathrm{WN}}(670 \mathrm{~nm})$ suggests that the MOBY-derived $\bar{g}(670 \mathrm{~nm})$ is biased high, as is indicated by the median satellite to in situ ratio of 1.719 for this band. In contrast, the NOMAD/ BOUSSOLE-derived $\bar{g}(670 \mathrm{~nm})$ results in a median ratio of only 1.091 for this band. $L_{W}(670 \mathrm{~nm})$ in open ocean waters is quite small given the strong attenuation by water at this wavelength, presenting a challenge for accurately estimating $L_{W}$ with in-water instrumentation. Most of the data in the NOMAD set were collected using free-fall radiometers, affording a much higher depth resolution of $L_{u}$ than is possible with the three fixed arms of MOBY. This increased depth resolution is beneficial to the extrapolation of $L_{u}$ to the surface, providing a better estimate of $L_{W}$ for wavelengths where light is rapidly attenuated by the water column.
It should be noted that 15 records in the NOMAD calibration data set are included in the validation data set, as both were derived from data within the SeaBASS archive. However, this represents only about $6 \%$ of the validation data set and, therefore, the validation data is largely independent of the NOMAD calibration data set.

\section{G. Untested Assumptions}

Several of the a priori requirements placed on in situ data for use in vicarious calibration (Table 1) cannot be addressed analytically with the data sets used in this study. However, comments on the adherence of these data sets to those requirements, as well as their overall relevance, can be made.

The requirement for a site with horizontally homogeneous water properties (Table 1 , item 3 ) is a practical requirement for any comparison between in situ data and a satellite-based measurement $[4,29]$. This requirement can be met with all in situ instrumentation and should not be seen as a limitation in the collection of in situ data for vicarious calibration.

The requirement that the in situ instrumentation undergo extraordinary instrument characterization and calibration to ensure low uncertainties in the radiometric measurements (Table 1 , item 5 ) can be met by the data sources employed for this study. Studies have shown that multiple sensor designs can have very similar and reproducible uncertainty budgets $[35,36]$ but, more importantly, the difficulty of making high-quality observations does not depend on calibrations alone. Buoy measurements have unique problems, not the least of which are discontinuous sampling at depth (as discussed in Section 5.F) and biofouling, that are easily overcome by using a profiling instrument or an above-water system. Any moored radiometer will suffer from biofouling during deployment, a limitation that does not affect ship-deployed in-water profilers or above-water systems [37].

The requirement for daily or weekly monitoring of derived $L_{\mathrm{WN}}$ (Table 1 , item 6) is not necessary for the vicarious calibration as implemented [4]. Current techniques for monitoring long-term stability of satellite-based ocean color instruments do not

Table 5. Validation Results ${ }^{a}$

\begin{tabular}{|c|c|c|c|c|c|c|c|c|c|}
\hline \multirow[b]{2}{*}{ Band } & \multirow[b]{2}{*}{$\mathrm{N}$} & \multicolumn{2}{|c|}{ MOBY } & \multicolumn{3}{|c|}{ msMOBY } & \multicolumn{3}{|c|}{ NOMAD/BOUSSOLE } \\
\hline & & Ratio & $\%$ Diff. & Ratio & $\%$ Diff. & Abs. UPD & Ratio & $\%$ Diff. & Abs. UPD \\
\hline$L_{\mathrm{WN}}(412)$ & 154 & 1.005 & 11.762 & 1.005 & 11.83 & 0.814 & 0.997 & 11.49 & 0.713 \\
\hline$L_{\mathrm{WN}}(443)$ & 236 & 0.938 & 15.96 & 0.936 & 16.10 & 0.324 & 0.924 & 16.32 & 0.313 \\
\hline$L_{\mathrm{WN}}(490)$ & 236 & 0.918 & 13.62 & 0.929 & 12.77 & 0.706 & 0.933 & 12.74 & 1.235 \\
\hline$L_{\mathrm{WN}}(510)$ & 127 & 0.953 & 11.97 & 0.948 & 12.01 & 0.815 & 0.985 & 12.26 & 1.636 \\
\hline$L_{\mathrm{WN}}(555)$ & 236 & 0.961 & 15.95 & 0.950 & 17.45 & 1.223 & 0.988 & 16.25 & 1.572 \\
\hline$L_{\mathrm{WN}}(670)$ & 233 & 1.719 & 87.21 & 1.218 & 81.18 & 12.69 & 1.091 & 85.49 & 15.42 \\
\hline$C_{a}$ & 383 & 1.001 & 27.80 & 0.988 & 27.90 & 1.569 & 1.060 & 29.42 & 3.636 \\
\hline
\end{tabular}

${ }^{a}$ Deep-water validation results for satellite data processed with the standard MOBY-derived $\overline{g_{\lambda}}$, the msMOBY-derived $\overline{g_{\lambda}}{ }^{\prime}$, and the weighted average of the NOMAD- and BOUSSOLE-derived $\overline{g_{\lambda}}$. The ratio column shows the median ratio of the satellite to in situ measured values; the \%Diff column shows the median percent difference for the same; and the Abs. UPD column shows the absolute unbiased percent difference between the satellite values estimated using the MOBY-derived $\overline{g_{\lambda}}{ }^{\prime}$ and the satellite values estimated from the alternative sea-truth data derived $\overline{g_{\lambda}}$. 


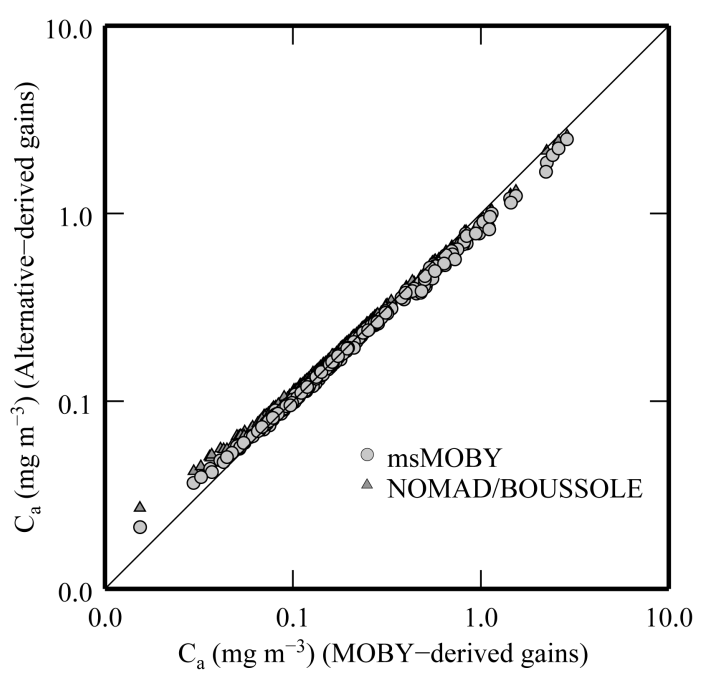

Fig. 7. Satellite-derived chlorophyll estimated from the two alternative $\bar{g}^{\prime}$ gain sets (msMOBY and NOMAD/BOUSSOLE) plotted versus the corresponding chlorophyll estimated from the standard MOBY $\bar{g}$

strictly rely on in situ data [14]. Modern sensors employ onboard calibration systems to track sensor stability or, as with SeaWiFS, use the Moon as an independent source for monitoring sensor stability $[13,38,39]$. Should future missions not have the same capability, it is conceivable that the alternative sources used in this study can comply with this requirement as well. Werdell et al. [6] demonstrated the feasibility of such an activity for historical sensors (e.g., the Coastal Zone Color Scanner). A large number of investigators equipped with suitable profilers (NOMAD) can also provide sufficient data over extended time periods, and COTS-based moorings (BOUSSOLE) can provide the same monitoring capabilities enjoyed with MOBY.

Avoidance of platform perturbations is a requirement (Table 1, item 7) that should be imposed on any radiance dataset and one easily satisfied by free-fall, profiling radiometers, which dominate the NOMAD data set. Also, it is rather easy to avoid platform shading and reflections with an above-water system if care is taken in the placement of the instrument and in its measurement protocol $[11,40]$.

Similarly, the requirement for a site with a high frequency of cloud-free days (Table 1 , item 8) may substantially increase the number of sea-truth data available for vicarious calibration; however, this requirement has no impact on the quality of the retrievals, merely on their frequency. When limited to a single source for sea-truth data, such a requirement is a statistical necessity; however, as has been demonstrated here, one need not be limited to a single source. A sufficient number of independent investigators could conceivably obtain enough sea-truth data to overcome any single site limitation due to cloud cover.

The collection of coincident measurements of atmospheric properties, such as aerosol optical thickness, (Table $\underline{1}$, item 9 ) was intended to provide a means for reducing the uncertainty in the selection of the aerosol models used by the atmospheric correction code in the vicarious calibration process. However, in practice, the vicarious calibration of the visible bands is determined after the calibration of the NIR bands. In fact, the two do not occur at the same geographic location. While coincident aerosol measurements have been used to evaluate the vicarious calibration of the NIR bands [41], they have not been used in the vicarious calibration of the visible band. To do so would violate the assumption of a vicarious calibration being a system-level calibration because a large part of the system is the aerosol selection mechanism for the atmospheric correction. Vicarious calibration of the visible bands assumes that the vicarious calibration of the NIR has been done and is accurate.

\section{Conclusions}

In this study, the use of alternative data sources for a vicarious calibration activity was examined and assumptions as to the necessary criteria for the collection of data to be used in vicarious calibration were tested. For a decade, the vicarious calibration of NASA's ocean color satellite missions have relied on a single source (MOBY) for ground-truth data. This approach has met the requirements for vicarious calibration data and successfully provided the ocean color community with a high-quality remotely sensed time series. Future sensors (e.g., VIIRS) may not have the benefit of a dedicated vicarious calibration activity such as MOBY. In addition, the need to rapidly achieve a stable calibration in an operational environment may necessitate more than a single calibration source. For example, with MOBY as the sole source, the SeaWiFS Project required three years to obtain sufficient sea-truth measurements for a stable vicarious calibration. Multiple data sources may have significantly reduced the time required to achieve a stable calibration (sample size of between 30 and 40 [4]).

Vicarious calibration coefficients derived from both the NOMAD and BOUSSOLE data sets are quite comparable to the standard MOBY-derived coefficients. The agreement between the multi- and hyperspectral MOBY results suggest that, in the absence of a hyperspectral radiance data source dedicated to the purpose of vicarious calibration, alternative, low-cost, and easily deployed instrumentation could serve as source data for the vicarious calibration of future missions. In the atmospheric correction inversion method to derive vicarious calibration coefficients, most of the components accounting for the majority of the TOA signal (i.e., atmospheric path radiance) do account for the full spectral response of the satellite radiometer $[4,16]$. Therefore, the requirement of hyperspectral resolution for the in situ observation may not be as necessary to minimize the overall uncertainty as was previously assumed.

The previously defined criteria for vicarious calibration data to be collected in low $C_{a}$ waters does not appear critical to the vicarious calibration pro- 
cess. Even without addressing the nonnegligible NIR radiance, data from waters with a $C_{a}$ concentration of up to $\sim 0.7 \mathrm{mg} \mathrm{m}^{-3}$ can be used without a detrimental impact on the retrieved coefficients. If the nonnegligible NIR radiance is appropriately removed, waters with $C_{a}$ concentrations as high as 1-3 $\mathrm{mg} \mathrm{m}^{-3}$ could be considered, as no bias was observed in the gains derived at higher $C_{a}$ concentrations. However, low $C_{a}$ waters do have the added benefit of helping to ensure that the requirement for horizontal homogeneity is met.

Restrictions on aerosol type and aerosol load also are not as critical as current criteria would suggest. These criteria were set to minimize the uncertainty in the atmospheric correction process. Figs. $\underline{5}$ and $\underline{6}$ suggest that the atmospheric correction algorithm adequately handles a wide variety of aerosol types and load, as long as the aerosol load is reasonably low (e.g. $\leq 0.2$ ). While only a small fraction of the available data had $\tau_{a}(865 \mathrm{~nm})$ greater than 0.20 , there was no trend in the $g_{\lambda}$ with increasing aerosol optical thickness. Likewise, there was no identifiable trend in the $g_{\lambda}$ with Angstrøm exponents, suggesting that the type of aerosol does not impact the gain, provided the satellite-retrieved aerosol model is appropriate for the atmospheric conditions present at the time of data collection. It appears that the largest uncertainties in the vicarious calibration process are not associated with the atmospheric correction process or the in situ instrumentation, but rather with the natural environment. This is supported by the lack of any trends with aerosol optical thickness or Ångstrøm exponents, as well as by the comparison between the variability in the MOBY-derived gains and those derived by the alternate target data sets (Table 2).

The vicarious calibration is performed with TÖA radiances, of which $L_{W}$ contributes no more than $\sim 10-15 \%$ under typical ocean conditions. Therefore, $85-90 \%$ of the uncertainty in the estimation of the vicarious calibration coefficients is not affected by the uncertainties on the in situ $L_{W}$ measurements. Using well-vetted deployment and processing protocols, in situ measured $L_{W}$ values can achieve an uncertainty on the order of 3-5\%, with lower uncertainties possible [36]. Brown et al. report uncertainties for MOBY on the order of $5 \%$. This, however, was prior to the implementation of a self-shading correction. Were such a correction applied, the uncertainty for MOBY may be reduced to $\sim 3 \%$ [42]. The MOBY data used in this study did not have such a correction applied. The uncertainties for the NOMAD and BOUSSOLE data sets appear to be of the same order as MOBY, as comparable measurement uncertainty is evident in the comparison of the retrieved $\overline{g_{\lambda}}{ }^{\prime}$ from the alternative sea-truth sources to the $\overline{g_{\lambda}{ }^{\prime}}$ obtained with MOBY.

It has been demonstrated that some requirements placed on the collection of in situ data for use in vicarious calibration are unnecessarily rigid. A number of the key requirements, specifically those for low chlorophyll waters, low aerosol atmosphere, and radiometric resolution sufficient to match the spectral response functions for the satellite instrument to be calibrated, can be relaxed without seriously compromising the calibration effort.

The authors thank the MOBY Operations Team, the BOUSSOLE project, and the numerous investigators who supplied data to the SeaBASS archive, which allowed for the creation of the NOMAD data set. This work would not have been possible without the generosity of these investigators. Special thanks go to Jim Brown of the University of Miami for his assistance in creating the msMOBY data set from the MATLAB binary files provided by the MOT. The BOUSSOLE project was supported and funded by the Centre National de la Recherche Scientifique (CNRS), the Institut National des Sciences de l'Univers (INSU), the French Space Agency (CNES), the European Space Agency (ESA, through ESTEC contract $14393 / 00 / \mathrm{NL} / \mathrm{D} . \mathrm{C}$. and ESRIN contract $17286 / 03 / \mathrm{I}-\mathrm{OL}$ ), and the U.S. National Aeronautics and Space Administration (NASA) through a letter of agreement with the Université Pierre et Marie Curie, Paris 6.

\section{References}

1. H. R. Gordon, "In-orbit calibration strategy for ocean color sensors," Remote Sens. Environ. 63, 265-278 (1998).

2. D. K. Clark, H. R. Gordon, K. J. Voss, Y. Ge, W. W. Broenkow, and C. Trees, "Validation of atmospheric correction over the oceans," J. Geophys. Res. 102, 17,209-17,217 (1997).

3. D. K. Clark, M. A. Yarbrough, M. Feinholz, S. Flora, W. Broenkow, Y. S. Kim, B. C. Johnson, S. W. Brown, M. Yuen, and J. L. Mueller, "MOBY, a radiometric buoy for performance monitoring and vicarious calibration of satellite ocean color sensors: measurement and data analysis protocols," NASA Tech. Memo. 2004-211621, NASA, Goddard Space Flight Center, Greenbelt, MD (2003).

4. B. A. Franz, S. W. Bailey, P. J. Werdell, and C. R. McClain, "Sensor-independent approach to the vicarious calibration of satellite ocean color radiometry," Appl. Opt. 46, 5068-5082 (2007).

5. C. Welsch, H. Swenson, S. A. Cota, F. DeLuccia, J. M. Haas, C. Schueler, R. M. Durham, J. E. Clement, and P. E. Ardanuy, "VIIRS (Visible Infrared Imager Radiometer Suite): a next generation operational environmental sensor for NPOESS," in Proceedings of IEEE Geoscience and Remote Sensing Symposium (IEEE, 2001), pp. 1020-1022.

6. P. J. Werdell, S. W. Bailey, B. A. Franz, A. Morel, and C. R. McClain, "On-orbit vicarious calibration of ocean color sensors using an ocean surface reflectance model," Appl. Opt. 46, 5649-5666 (2007).

7. R. A. Barnes, J. Robert, E. Eplee, W. D. Robinson, G. M. Schmidt, F. S. Patt, S. W. Bailey, M. Wang, and C. R. McClain, "The calibration of SeaWiFS on orbit," Proc. SPIE 4135, 281-293 (2000).

8. J. L. Mueller, R. W. Austin, A. Morel, G. S. Fargion, and C. R. McClain, "Ocean Optics Protocols for Satellite Ocean Color Sensor Validation, Revision 4: Introduction, background and conventions," NASA Tech. Memo. 2003-211621, NASA, Goddard Space Flight Center, Greenbelt, MD (2003).

9. P. J. Werdell and S. W. Bailey, "An improved in-situ bio-optical data set for ocean color algorithm development and satellite data product validation," Remote Sens. Environ. 98, 122140 (2005).

10. D. Antoine, J.-F. Desté, G. Bécu, F. Louis, A. J. Scott, and P. Bardey, "The BOUSSOLE buoy: A new transparent-to-swell 
taut mooring dedicated to marine optics: design, tests and performance at sea," J. Atmos. Ocean. Technol. (to be published).

11. G. Zibordi, S. B. Hooker, J.-F. Berthon, and D. D'Alimonte, "Autonomous above-water radiance measurements from an offshore platform: A field assessment experiment," J. Atmos. Ocean. Technol. 19, 808-819 (2002).

12. P.-Y. Deschamps, B. Fougnie, R. Frouin, P. Lecomte, and C. Verwaerde, "SIMBAD: A field radiometer for satellite ocean-color validation," Appl. Opt. 43, 4055-4069 (2004).

13. R. A. Barnes, R. E. Eplee Jr., G. M. Schmidt, F. S. Patt, and C. R. McClain, "Calibration of SeaWiFS. I. Direct techniques," Appl. Opt. 40, 6682-6700 (2001).

14. B. A. Franz, P. J. Werdell, G. Meister, S. W. Bailey, R. E. Eplee, G. C. Feldman, E. Kwiatkowska, C. R. McClain, F. S. Patt, and D. Thomas, "The continuity of ocean color measurements from SeaWiFS to MODIS," Proc. SPIE 5882, 58820W (2005)

15. R. A. Barnes, A. W. Holmes, W. L. Barnes, W. E. Esaias, and T. Svitek, "SeaWiFS prelaunch radiometric calibration and spectral characterization," NASA Tech. Memo. 104566, NASA, Goddard Space Flight Center, Greenbelt, MD (1995).

16. H. R. Gordon, "Remote sensing of ocean color: a methodology for dealing with broad spectral bands and significant outof-band response," Appl. Opt. 34, 8363-8374 (1995).

17. M. Wang, B. A. Franz, R. A. Barnes, and C. R. McClain, "Effects of spectral bandpass on SeaWiFS-retrieved near-surface optical properties of the ocean," Appl. Opt. 40, 343-348 (2001).

18. F. S. Patt, R. A. Barnes, R. E. Eplee Jr., B. A. Franz, W. D. Robinson, G. C. Feldman, S. W. Bailey, J. Gales, P. J. Werdell, M. Wang, R. Frouin, R. P. Stumpf, R. A. Arnone, J. R. W. Gould, P. M. Martinolich, V. Ransibrahmanakul, J. E. O'Reilly, and J. A. Yoder, "Algorithm updates for the fourth SeaWiFS data reprocessing," NASA Tech. Memo. 206892, Vol. 22, NASA, Goddard Space Flight Center, Greenbelt, MD (2003).

19. D. K. Clark, M. Feinholz, M. Yarbrough, B. C. Johnson, S. W. Brown, Y. S. Kim, and R. A. Barnes, "Overview of the radiometric calibration of MOBY," in Earth Observing Systems VI, Vol. 4483, W. L. Barnes, ed. (SPIE, 2002), pp. 64-76.

20. P. J. Werdell, S. W. Bailey, G. Fargion, C. Pietras, K. Knobelspiesse, G. Feldman, and C. McClain, "Unique data repository facilitates ocean color satellite validation," EOS Trans. Am. Geophys. Union 84, (2003).

21. D. Antoine, M. Chami, H. Claustre, F. d'Ortenzio, A. Morel, G. Bécu, B. Gentili, F. Louis, J. Ras, E. Roussier, A. J. Scott, D. Tailliez, S. B. Hooker, P. Guevel, J.-F. Dest', C. Dempsey, and D. Adams, "BOUSSOLE: a joint CNRS-INSU, ESA, CNES, and NASA ocean color calibration and validation activity," NASA Tech. Memo. 214147, NASA, Goddard Space Flight Center, Greenbelt, MD (2007).

22. M. Wang, "The Rayleigh lookup tables for the SeaWiFS data processing: accounting for the effects of ocean surface roughness," Int. J. Remote Sensing 23, 2693-2702 (2002).

23. M. Wang, "A refinement for the Rayleigh radiance computation with variation of the atmospheric pressure," Int. J. Remote Sensing 26, 5651-5653 (2005).

24. H. R. Gordon and M. Wang, "Retrieval of water-leaving radiance and aerosol optical thickness over the oceans with SeaWiFS: A preliminary algorithm," Appl. Opt. 33, 443-452(1994).

25. H. R. Gordon and M. Wang, "Influence of oceanic whitecaps on atmospheric correction of SeaWiFS," Appl. Opt. 33, 77547763 (1994).

26. R. Frouin, M. Schwindling, and P. Y. Dechamps, "Spectral reflectance of sea foam in the visible and near infrared: In situ measurements and remote sensing implications," J. Geophys. Res. 101, 14,361-14,371 (1996).

27. K. D. Moore, K. J. Voss, and H. R. Gordon, "Spectral reflectance of whitecaps: Their contribution to water-leaving radiance," J. Geophys. Res. 105, 6493-6499 (2000).
28. M. Wang, "Atmospheric correction of ocean color sensors: Computing atmospheric diffuse transmittance," Appl. Opt. 38, 451-455 (1999).

29. S. Bailey and P. Werdell, "A multi-sensor approach for the onorbit validation of ocean color satellite data products," Remote Sens. Environ. 102, 12-23 (2006).

30. A. Morel and L. Prieur, "Analysis of variations in ocean color," Limnol. Oceanogr. 22, 709-722 (1977).

31. D. A. Siegel, M. Wang, S. Maritorena, and W. Robinson, "Atmospheric correction of satellite ocean color imagery: the black pixel assumption," Appl. Opt. 39, 3582-3591 (2000).

32. J. E. O'Reilly, S. Maritorena, D. A. Siegel, M. C. O'Brien, D. Toole, B. G. Mitchell, M. Kahru, F. P. Chavez, P. Strutton, G. F. Cota, S. B. Hooker, C. R. McClain, K. L. Carder, F. E. Muller-Karger, L. W. Harding Jr., A. Magnuson, D. Phinney, G. F. Moore, J. Aiken, K. R. Arrigo, R. Letelier, and M. Culver, “Ocean color chlorophyll a algorithms for SeaWiFS, OC2, and OC4: Version 4," in SeaWiFS Postlaunch Calibration and Validation Analysis, Part 3, S. B. Hooker and E. R. Firestone, eds. (NASA Tech. Memo. 206892, Vol. 11, NASA, Goddard Space Flight Center, Greenbelt, Maryland, 2000), pp. 9-24.

33. W. L. Barnes, T. S. Pagano, and V. V. Salomonson, "Prelaunch characteristics of the Moderate Resolution Imaging Spectroradiometer (MODIS) on EOS-AM 1," IEEE Trans. Geosci. Remote Sens. 36, 1088-1100 (1998).

34. S. B. Hooker, W. E. Esaias, G. C. Feldman, W. W. Gregg, and C. R. McClain, "An overview of SeaWiFS and ocean color," NASA Tech. Memo. 104566, NASA, Goddard Space Flight Center, Greenbelt, MD (1992).

35. S. B. Hooker, S. McLean, J. Sherman, M. Small, and G. Zibordi, "The Seventh SeaWiFS Intercalibration Round-Robin Experiment (SIRREX-7)," SeaWiFS Postlaunch Technical Report Series 2000-206892, NASA, Goddard Space Flight Center, Greenbelt, MD (1999).

36. S. B. Hooker and S. Maritorena, "An evaluation of oceanographic radiometers and deployment methodologies," J. Atmos. Ocean. Technol. 17, 811-830 (2000).

37. J. L. Mueller, D. K. Clark, V. S. Kuwahara, G. Lazin, S. Brown, G. S. Fargion, M. A. Yarbrough, M. Feinholz, S. Flora, W. Broenkow, Y. S. Kim, B. C. Johnson, M. Yuen, P. G. Strutton, T. D. Dickey, M. R. Abbott, R. M. Letelier, M. R. Lewis, S. McLean, F. P. Chavez, J. R. M. A. Barnard, A. Subramaniam, D. Manov, X. Zheng, J. L. W. Harding, R. A. Barnes, and K. R. Lykke, "Ocean Optics Protocols for Satellite Ocean Color Sensor Validation, Revision 4: Special topics in ocean optics protocols and appendices," NASA Tech. Memo. 2004-211621, NASA, Goddard Space Flight Center, Greenbelt, MD (2003).

38. J. Sun, X. Xiong, B. Guenther, and W. Barnes, "Radiometric stability monitoring of the MODIS reflective solar bands using the moon," Metrologia 40, S85-S88 (2003).

39. F. S. Patt, R. E. Eplee, R. A. Barnes, G. Meister, and J. J. Butler, "Use of the moon as a calibration reference for NPP VIIRS," Proc. SPIE 5882, 588215 (2005).

40. S. B.Hooker, G. Lazin, G.Zibordi, and S. McLean, "An evaluation of above- and in-water methods for determining water-leaving radiances," J. Atmos. Ocean. Technol. 19, 486-515 (2002).

41. G. Fargion, R. Barnes, and C. R. McClain, "In situ aerosol optical thickness collected by the SIMBIOS Program (19972000): Protocols, and data QC and analysis," NASA Tech. Memo. 209982, NASA, Goddard Space Flight Center, Greenbelt, MD (2001).

42. S. W. Brown, S. J. Flora, M. E. Feinholz, M. A. Yarbrough, T. Houlihan, D. Peters, Y. S. Kim, J. L. Mueller, B. C. Johnson, and D. K. Clark, "The Marine Optical BuoY (MOBY) radiometric calibration and uncertainty budget for ocean color satellite sensor vicarious calibration," Proc. SPIE 6744, 67441M (2007). 